\title{
Weighted Polynomial Approximation on the Integers
}

By

L. A. RUBEL $\left.{ }^{1}\right)$ and B. A. TAYLOR

We prove here some polynomial approximation theorems, somewhat related to the Szasz-Müntz theorem, but where the domain of approximation is the integers, by dualizing a gap theorem of C. RÉnY for periodic entire functions. In another paper [7], we shall prove, by similar means, a completeness theorem for some special sets of entire functions.

It is well known (see, for example [1]) that if $E$ is the space of all entire functions in the topology of uniform convergence on compact sets, then the dual space of continuous complex-valued linear functionals on $E$ may be represented as $E_{0}$, the space of entire functions of exponential type. Now let $E(1)$ be the space of entire functions of period 1. Then it may be shown that the dual of $E(1)$ can be represented as $E_{0}(1)$, where $E_{0}(1)$ is the following quotient space of $E_{0}$ : define $f \sim g$ for functions $f, g \in E_{0}$ if $f-g$ is a multiple of $\sin \pi z$, and let $E_{0}(1)$ be the space of equivalence classes of $E_{0}$ modulo this relation of equivalence. Now $E_{0}(1)$ is apparently the same space as the space of restrictions of functions in $E_{0}$ to the integers $Z$. Fach such restriction is just a two-sided sequence of complex numbers, of at most exponential growth. Conversely, it is easy to interpolate any such sequence by an entire function of exponential type. Thus, the dual of $E(1)$ is just the space of all such sequences. Actually, we establish this identification by another procedure.

To any theorem about periodic entire functions will correspond a theorem about the space of sequences described above. In [6], C. RÉNY I proved an interesting gap theorem, reproduced below. We show by means of duality that certain theorems of polynomial approximation are equivalent to this theorem. The domain of approximation is the integers in one case, and the positive integers in another case. To our knowledge, the problem of polynomial approximation on the integers has not been considered except in the note [3]. We know of no direct proof of our results. Ultimately, the RÉNYI result depends on a simple application of Rolle's theorem. It would be of interest to have more precise gap theorems than the RENYI theorem and also to have direct proofs of the results we prove by means of it.

'Theorem (C. Rényi). Let $F(z)$ be a periodic entire function such that $F(0)=0$ and $F^{(n)}(0)=0$ for $n \in N$, where $N$ is a set of positive integers of lower density exceeding

1) The research of the first author was partially sponsored by a grant from the United States Air Force Office of Scientific Research, Grant Number AFOSR 460-63. 
$1 / 2$, in the sense that $\lim \inf r^{-1} N(r)>1 / 2$ as $r \rightarrow \infty$, where $N(r)$ is the number of elements of $N$ that do not exceed $r$. Then $F$ must be the null function $F=0$.

We shall use the following notation. Let $\Gamma$ be the collection of all two-sided sequences $b=\left\{b_{k}\right\}, k=0, \pm 1, \pm 2, \ldots$ of complex numbers $b_{k}$ of at most exponential growth; that is $\left|b_{k}\right| \leqq A \exp (B|k|)$ for some constants $A$ and $B$. Let $I_{+}$be the collection of one-sided sequences $d=\left\{d_{k}\right\}, k=0,1,2, \ldots$, of complex numbers $d_{k}$ of at most exponential growth. If $p$ is any polynomial, then $\left.p\right|_{\boldsymbol{z}}$ (respectively $\left.\left.p\right|_{\mathbf{z}_{+}}\right)$belongs to $I$ (respectively to $\Gamma_{+}$). We call such elements of $\Gamma$ and $\Gamma_{+}$polynomials.

By $N$, we denote any collection of non-negative integers, with 0 adjoined. We shall stress throughout the paper that $0 \in N$. By $P_{N}$ (respectively $P_{N}^{+}$) we denote the collection of all polynomials in $\Gamma$ (respectively $\Gamma_{+}$) with exponents lying in $N$, that is

$$
p(x)=\sum a_{n} x^{n}, \quad a_{n}=0 \text { for } n \notin N .
$$

We shall be concerned with finding conditions on $N$ such that $P_{N}$ be dense in $I$ (respectively that $P_{N}^{+}$be dense in $\Gamma_{+}$) where $\Gamma$ and $\Gamma_{+}$are given appropriate topologies. The topologies we introduce are familiar from the theory of sequence spaces. Denote by $\Gamma^{*}$ (respectively $\Gamma_{+}^{*}$ ) the collection of all sequences $a=\left\{a_{k}\right\}, k=0, \pm 1, \pm 2, \ldots$ (respectively $c=\left\{c_{k}\right\}, k=0,1,2, \ldots$ ) such that $\sum\left|a_{k} b_{k}\right|<\infty$ for each $b \in I$ (respectively such that $\sum\left|c_{k} d_{k}\right|<\infty$ for each $\left.d \in \Gamma_{+}\right)$. It is easy to verify that $a \in I^{*}$ if and only if $\left|a_{k}\right|^{1 / k} \rightarrow 0$ as $|k| \rightarrow \infty$, with a similar statement for $\Gamma_{+}^{*}$. For each $a \in I^{*}$, the mapping \|\|$_{a}: I \rightarrow \mathbf{R}$ of $I$ into the real numbers $\mathbf{R}$, given by

$$
\|b\|_{a}=\sum\left|b_{k} a_{k}\right|
$$

is a seminorm on $I$. The collection of all such seminorms, as $a$ varies over $T^{*}$, determines a locally convex topology on $\Gamma$, the so-called normal topology [4, p. 410]. A similar procedure gives a corresponding topology on $\Gamma_{+}$, and we shall suppose from now on that $\Gamma$ and $\Gamma_{+}$are equipped with these topologies. We may now state our approximation results.

'Theorem 1. If $N$ has lower density greater than $1 / 2$, then $P_{N}$ is dense in $\Gamma$.

Theorem 2. If $N$ contains a set of even (respectively add) integers, of positive lower density, then $P_{N}^{+} i$ s dense in $\Gamma_{+}$.

We prove these results by showing that they are equivalent to RÉryi's theorem. We shall require three well-known preliminary results.

Proposition 1. There is a linear one:one correspondence between $\Gamma^{*}$ and the collection of periodic entire functions of period $2 \pi i$ given as follows. Let $F(z)$ be such a function. Then

$$
F(z)=\sum_{-\infty}^{\infty} a_{k} e^{k z}
$$

where

$$
a_{k}=\frac{1}{2 \pi i} \int_{0}^{2 \pi i} F(z) e^{-k z} d z
$$


Furthermore,

$$
\left|\alpha_{k}\right|^{1 /|k|} \rightarrow 0 \text { as }|k| \rightarrow \infty,
$$

so that the series in (1) converges absolutely and uniformly on every compact set. Conversely, for each sequence $\left\{a_{k}\right\}$ such that (3) holds, the expression (1) defines an entire function of period $2 \pi i$.

Proposition 2. The pairing of $\Gamma$ and $\Gamma^{*}$ defined by

$$
\langle b, a\rangle=\sum b_{k} a_{k}, \quad b \in \Gamma, \quad a \in \Gamma^{*}
$$

establishes $\Gamma^{*}$ as the topological dual space of $I$.

Proposition 3. The pairing of $\Gamma_{+}$and $I_{+}^{*}$ defined by

$$
\langle d, c\rangle=\sum d_{k} c_{k}, \quad d \in \Gamma_{+}, \quad c \in \Gamma_{+}^{*}
$$

establishes $\Gamma_{+}^{*}$ as the topological dual space of $I_{+}$.

Proposition 1 may be found in [8, p.361]. Propositions 2 and 3 are easy to verify and are given as an example in $[4$, p. 424]. We shall now prove that Theorem 1 is equivalent to Rény's theorem. From Proposition 1, we know that an entire function $F$ of period $2 \pi i$ is of the form $F^{\prime}(z)=\sum a_{k} \exp (k z)$ for some $a=\left\{a_{k}\right\}$ in $I^{*}$. Hence

$$
F^{(n)}(0)=\sum_{-\infty}^{\infty} a_{k} k^{n}, \quad n=0,1,2, \ldots,
$$

using the convention that $0^{0}=1$. Consequently, Résy's theorem is equivalent to the following result.

Proposition 4. Suppose that $N$ has lower density greater than $1 / 2$ and that $0 \in N$. If $a=\left\{a_{k}\right\} \in \Gamma^{*}$ and if

$$
\sum_{-\infty}^{\infty} a_{k} k^{n}=0 \quad \text { for each } \quad n \in N
$$

then $a_{k}=0$ for $k=0, \pm 1, \pm 2, \ldots$.

Now it is easy to sce that Theorem 1 is also equivalent to Proposition 4. For by the Hahn-Banach theorem, $P_{N}$ is dense in $\Gamma$ if and only if the only continuous linear functional on $\Gamma$ which annihilates $P_{N}$ is the zero functional. By Proposition 2, each continuous linear functional $L$ on $\Gamma$ is of the form $L(b)=\langle b, a\rangle$ for some $a \in \Gamma^{*}$. Moreover, the continuous linear functional determined by $a \in \Gamma^{*}$ annihilates $P_{N}$ if and only if $\sum a_{k} k^{n}=0$ for each $n \in N$. However $a \in \Gamma^{*}$ represents the zero functional if and only if $a_{k}=0, k=0, \pm 1, \pm 2, \ldots$, and the proof is done.

We shall now show that Theorem 2 is equivalent to RÉxyI's theorem. Our first step is to deduce the following two results from RÉNYI's theorem.

Proposition 5. If $F$ is an even periodic entire function and if $F^{(n)}(0)=0$ for every $n$ in a collection $N$ of even nonnegative integers, with $0 \in N$, such that $N$ has positive lower density, then $F$ must be the null function $F=0$.

Proposition 6. If $F$ is an odd periodic entire function, and if $F^{(n)}(0)=0$ for each $n$ in a collection $N$ of odd nonnegative integers, such that $N$ has positive lower density, then $F$ must be the null function $F=0$. 
'These results follow casily from the RExy theorem since the union of the odd (respectively even) positive integers with a set of even (respectively odd) integers of positive lower density must have lower density exceeding 1/2. Now Proposition 5 und Proposition 6 together imply Rénys's theorem as we see on writing $F=F_{1}+F_{2}$ where $F_{1}(z)=\frac{1}{2}(F(z)+F(-z)), F_{2}(z)=\frac{1}{2}(F(z)-F(-z))$. We now prove that Proposition 5 is equivalent to Proposition 7 below and that Proposition 6 is equivalent to Proposition 8 below.

Proposition 7. If $c=\left\{c_{k}\right\}$ belongs to $\Gamma_{+}$and if $\sum c_{k} k^{n}=0$ for $n$ in a collection $N$, with $0 \in N$, of even nonnegative integers, and of positive lower density, then $c_{k}=0$ for $k=0,1,2, \ldots$.

Proposition 8. If $c=\left\{c_{k}\right\}$ belongs to $I_{+}$and if $\sum c_{k} k^{n}=0$ for $n$ in a collection $N$ of odd positive integers, except that $0 \in N$, and of positive lower density, then $c_{k}=0$ for $k=0,1,2, \ldots$.

To prove that Propositions 5 and 7 are equivalent, let $c=\left\{c_{k}\right\}$ belong to $\Gamma_{+}$and define

$$
F(z)=\sum_{k=0}^{\infty} c_{k}\left(e^{k z}+e^{-k z}\right)
$$

Then $F$ is an even periodic entire function. Furthermore, if $n$ is even and positive, then

$$
F^{\prime}(n)(0)=2 \sum_{k=1}^{\infty} c_{k} k^{n}
$$

so that the equivalence is clear. A similar proof shows that Propositions 6 and 8 are equivalent.

It remains only to prove that Theorem 2 is equivalent to Propositions 7 and 8 together. This follows from the Hahn-Banach Theorem, by the same argument as that given after Proposition 4.

An interesting question is whether the hypothesis on the parity of the elements of $N$ can be dropped from Theorem 2 . (It is easy to construct sets of nonnegative integers of positive lower density whose even and odd parts have lower density zero.) An investigation of this question could perhaps lead to a new proof of Riviri's theorem.

\section{References}

[1] L. Eurenireis, Mean periodic functions. Amer. J. Math. 77, 293-328 (1955).

[2] V. Ganapathy IYer, On the space of integral functions I. J. Indian Math. Soc. 12, 13-30 (1948).

[3] P. Koosis, L'approximation ponderée sur des progressions arithmétiques d'intervalles ou de points. C.R. Acad. Sci. Paris 261, $3022-3024$ (1965).

[4] G. KӧтнE, Topologische Lineare Räume. Berlin-Göttingen-Heidelberg 1960.

[5] C. Rény i, On a conjecture of Pólya. Acta Math. Acad. Sci. Hungar. 7, 145-149 (1956).

[6] C. Rinsy I, On periodic entire functions. Acta Math. Acad. Sci. Hungar. 8, 227-233 (1957).

[7] I. A. RubEl and B. A. TaYLoR, A completeness theorem for entire functions. J. Indian Math. Soc. (to appear).

[8] S. Saks and A. Zyamund, Analytic Functions. 2nd ed., Warsaw 1965. 
Anschrift der Autoren:

L. A. Rubel

Department of Mathematics

B. A. Taylor

University of Illinois

Department of Mathematics

Urbana (Ill.), USA

University of Michigan

Ann Arbor (Mich.), USA 